\section{ANTHRAX: AN OLD DISEASE RAISING NEW FEARS}

Anthrax is a potentially fatal bacterial infection caused by the aerobic, Gram-positive rod Bacillus anthracis. It is a disease of great historical interest, which has recently been the subject of many headlines as a result of its potential use as a biological weapon. Anthrax is primarily a disease of herbivores, which are exposed to spores in the soil while grazing. The disease is most prevalent among domestic herbivores such as cattle, sheep, horses, and goats. The distribution of anthrax is worldwide. In Canada, "anthrax zones" include the western Prairies, northern Alberta, and the Northwest Territories (1). In North America, veterinary vaccination programs have drastically reduced the number of outbreaks. Anthrax continues to be endemic however, in regions of Africa, Central Asia, Spain, Greece, Turkey, Albania, Romania (2). Infection in humans develops when spores of $B$. anthracis enter the body through a skin abrasion, via ingestion, or inhalation. Ninety-five percent of human cases of anthrax are the cutaneous form and are the most often the result of contact with infected animals or animal products in an agricultural or industrial setting. Gastrointestinal anthrax, resulting from the ingestion of viable spores, is exceedingly rare. Approximately 5\% of human anthrax cases are of the inhalational type.

\section{History}

Anthrax was first described over 3500 years ago. It is believed to have been responsible for one of the great plagues in ancient Egypt and cases were recorded by the ancient Romans (3). The anthrax bacillus was the organism used by Robert Koch in the development of his postulates and is considered the first "germ" to be proven to cause human disease (4). More recently, $B$. anthracis has been the organism of choice experiments of biological weapons. In 1941 the British released anthrax spores on Gruinard Island off the coast of Scotland. Spores capable of infection survived for 45 years until the island was decontaminated with formaldehyde and seawater in 1986 (5). The United States experimented with anthrax in the 1950s and 1960s until the program was stopped by Richard Nixon in the 1970 (6). In 1979, a large outbreak of anthrax in the former Soviet Union at Sverdlovsk resulted in the deaths of dozens of people. Though autopsy results were confiscated by the government, the source of the infections is thought to have been a nearby military microbiology plant (7).

\section{Pathogenesis}

Capsular polypeptide and anthrax toxin are the primary virulence factors of $B$. anthracis. Anthrax toxin consists of three proteins (8) including protective antigen (PA), edema factor (EF), and lethal factor (LF). PA binds to plasma membranes of target cells where it is cleaved by a cellular protease into two fragments. The larger of the two fragments remains bound to the cell surface and serves as a receptor that mediates endocytic enter of EF and LF into the cell. EF serves to increase intracellular cyclic adenosine monophosphate (cAMP), which, ultimately results in the massive edema seen in anthrax patients. At high doses LF causes lysis of macrophages. At lower doses it serves to increase expression of tumour necrosis factor (TNF) and interleukin-1 (IL-1). It is believed as infection progresses, the threshold for lysis is reached causing a massive release of inflammatory mediators leading to shock and death (6).

\section{Clinical Manifestations}

Cutaneous anthrax occurs when spores of $B$. anthracis are introduced into the skin. Within hours, the spores begin to germinate and release anthrax toxin. Soon thereafter, a small red macule appears at the site of inoculation. The lesion progresses to a papular stage and within 24 to 48 hours, the papules enlarge and become vesicular. The lesion ruptures during the first week and forms an ulcer encircled by a black eschar and surrounded by edema out of proportion to the size of the ulcer. The fully developed lesion is usually painless. In mild cases, the patient is afebrile with no constitutional symptoms, however, in more severe infections, associated features include fever, malaise, and regional adenopathy (9). Eighty to ninety percent of untreated cases undergo spontaneous healing. Ten to twenty percent of untreated cases lead to a bacteremia and death.(8) If recognized and treated promptly, cutaneous anthrax is very rarely fatal.

Gastrointestinal anthrax usually results from the ingestion of undercooked meat of infected animals. Bacteria are transported to mesenteric and regional lymph nodes leading to hemorrhagic adenitis, ascites, 
and bacteremia. The patient presents with variable symptoms including severe abdominal pain, fever, nausea and vomiting, and bloody diarrhea. The pharynx may also be infected causing ulcers and edema of the neck, occasionally leading to airway compromise. Early diagnosis of gastrointestinal anthrax is difficult resulting in a high mortality rate (9).

Inhalational anthrax has historically occurred among wool workers and those closely associated with infected animals. It is aerosolized anthrax that is most lethal and has potential use as a biological weapon. Initial symptoms may present up to 6 weeks after exposure and closely resemble a severe viral respiratory disease, making early detection difficult. Accordingly, a high degree of suspicion is required to correctly diagnose this condition. Inhalational anthrax is not considered a true pneumonia. Though the 1 to $2 \mathrm{~mm}$ spores are an ideal size for alveolar deposition, the spores are engulfed by alveolar macrophages and transported to the mediastinal and peribronchial lymph nodes. The anthrax bacilli multiply in the lymph nodes and spread throughout the body and blood(10). The infected patient initially presents with fever, non-productive cough, myalgia, and malaise. Radiographs show a classic widened mediastinum, which is evidence of hemorrhagic mediastinitis and pleural effusions. One to three days after the onset of symptoms, the disease enters a rapid, fulminant course consisting of dyspnea, strident cough and chills, and culminates in death (10). The mortality of inhalational anthrax approaches $100 \%$ and treatment is rarely successful (8). The number of spores required for infection is not known. The U.S. Department of Defence estimates the number to be between 8000 and 10000 spores (11).

\section{Diagnosis}

In cutaneous anthrax, the ulcerative eschar must be differentiated from other causes of papular lesions causing lymphadenopathy. The most likely cause of such lesions is staphylococcal lymphadenitis (10). When in an endemic anthrax region, and the ingestion of contaminated meat is suspected, symptoms of an acute abdomen should be considered to possibly be the early signs of intestinal anthrax infection (10). Diagnosis of inhalational anthrax in the early stage is difficult because of the similarity of the presenting symptoms to a viral respiratory tract infection. The classic widened mediastinum on radiograph may help in diagnosis but must be differentiated from noninfectious causes such as superior vena cava syndrome or a dissecting or ruptured aortic aneurysm. The widened mediastinum may also be seen in an acute bacterial mediastinitis and fibrous mediastinitis due to Histoplasma capsulatum (10).
Regardless of the suspected route of infection, Gram staining of pertinent body fluids should be done to determine the causative organism. Serologic diagnosis may be made through a microhemagglutination test for the PA component of the anthrax toxin. Diagnosis may also be made by specific enzyme-linked immunosorbent assays (ELISA).

\section{Prevention and Treatment}

The anthrax vaccine is an inactivated, cell free product given in 6 doses (12). It is recommended for military personnel, individuals closely associated with potentially infected animals, and researchers who may be in contact with anthrax spores. It is not currently licensed for use in Canada but may be obtained through Health Canada's special access program (12). There are safety issues that have limited the use of the vaccine in humans. It has been associated with edema and necrosis at the site of injection and rarely death (6). Few comparative studies have examined the efficacy of human anthrax vaccines. A review of two studies (a 1962 US study using an inactivated vaccine similar to the US vaccine of today and a 1976 Russian study using a live attenuated vaccine) concluded that it carries an overall efficacy of $84 \%$ in preventing anthrax infection (13). A newer examination of the efficacy of the current vaccine is required. The ability of the vaccine to provide protection from aerosolized anthrax, such as in the form of a biological weapon, has not been tested and remains unknown (10).

B. anthracis is susceptible to penicillin, amoxicillin, chloramphenicol, doxycycline, erythromycin, streptomycin, and ciprofloxacin. Intravenous administration is recommended in cases of inhalational and gastrointestinal anthrax as well as in severe cutaneous infections (10). It should be considered resistant to third generation cephalosporins, cefuroxime, sulfamethoxazole, and trimethoprim. Of particular concern in bioterrorism is the fact that it is possible to manufacture anthrax to be resistant to penicillin. When biological warfare is suspected, ciprofloxacin is the drug of choice (14).

\section{Summary}

The history of anthrax and that of human agricultural development have been intertwined for thousands of years. In the present time, new fears have arisen of the use of $B$. anthracis as a biological weapon. Rapid recognition of the signs and symptoms of an anthrax infection is essential if antibiotic treatment is to be effective. This is especially true with inhalational anthrax. Rapid diagnosis of inhalational anthrax is made difficult by the non-specific nature of the initial presenting illness. A high degree of suspicion is 
required to make a timely diagnosis. Health care professionals must have an understanding of the clinical presentation, the pathophysiology, and treatment of anthrax infection.

\section{Shaun Morris}

Class of 2003, School of Medicine

Queen's University

\section{REFERENCES}

1. Dragon, DC, Elkin, BT, Nishi, JS, Ellsworth, TR. A review of anthrax in Canada and its implications for research of the disease in northern bison [review]. Journal of Applied Microbiology 87: 208-213; 1999.

2. Hugh-Jones, M. 1996-1997 global anthrax report. Journal of Applied Microbiology 87: 189-191; 1999.

3. Dirckx, JH. Virgil on anthrax. American Journal of Dermatopathology 3: 191-195; 1981.

4. Zydowicz, D. Anthrax: a disease from antiquity visits the modern world. Minn Med 81: 19-20; 1998.

5. Christophe, GW, Cieslack, TJ, Pavlin, JA, et al. Biological warfare: a historical perspective. JAMA 278: 412-417; 1997.
6. Shafazand, S, Doyle, R, Ruoss, S, Weinacker, A, Raffin, TA Inhalational Anthrax. Chest 116: 1369-1376; 1999.

7. Meselson, M, Guillemin, J, Hugh-Jones, M, et al. The Sverdlovsk anthrax outbreak of 1979. Science 266: 1202-1208; 1994.

8. Harrisons Principles of Internal Medicine, 14th Edition. Editors: Fauci, AS, Braunwald, E, Isselbacher, KJ, Wilson, JD, Martin, JB, Kasper, DL, Hauser, SL, Longo, DL. New York, New York: The McGraw-Hill Companies, Inc, 1998.

9. LaForce, FW. Anthrax. Clinical Infectious Diseases. 19: 10091014; 1994.

10. Dixon, TC, Meselson, M, Guillemin, J, Hanna, PC. Anthrax [review]. New England Journal of Medicine 341: 815-26; 1999

11. Franz, DR, Jahrling, PB, Friedlander, AM, et al. Clinical recognition and management of patients exposed to biological warfare agents. JAMA 278: 399-411; 1997.

12. Weir, E. Anthrax: of bison and bioterrorism. CMAJ 163(5): 608; 2000 Sept 5.

13. Jefferson, T, Demicheli, V, Deeks, J, Graves, P, Pratt, M, Rivetti, D. Vaccines for preventing anthrax [Cochrane review]. In: The Cochrane Library; Issue 3, 2000. Oxford: Update Software.

14. Inglesby TV, Henderson, DA, Bartlett, JG, et al. Anthrax as a biological weapon: medical and public health management [review]. JAMA 281: 1735-1745; 1999. 